\title{
COMMENTS
}

Comments are short papers which criticize or correct papers of other authors previously published in the Physical Review. Each Comment should state clearly to which paper it refers and must be accompanied by a brief abstract. The same publication schedule as for regular articles is followed, and page proofs are sent to authors.

\section{Comment on "Algebraic perturbation theory for polar fluids: A model for the dielectric constant"}

\author{
István Szalai* \\ Department of Chemistry, The University of Hong Kong, Pokfulam Road, Hong Kong, Republic of China \\ Kwong-Yu Chan ${ }^{\dagger}$ \\ Department of Chemistry, The University of Hong Kong, Pokfulam Road, Hong Kong, Republic of China \\ Douglas Henderson ${ }^{*}$ \\ Department of Chemistry and Biochemistry, Brigham Young University, Provo, Utah 84602-5700
}

(Received 19 October 1999)

Kalikmanov [Phys. Rev. E 59, 4085 (1999)] proposed a perturbation theory method to calculate the dielectric constant of dipolar hard sphere fluids using an infinitely long cylindrical container to avoid the depolarization. We demonstrate that while the method is very helpful, his theory appears to be incomplete because of the incorrect calculation of the corresponding three-body integrals. It is shown that with the correct consideration of these terms the theory is consistent with the results of earlier work in low-density limit, and at high densities the method yields the equation of Tani et al. [Mol. Phys. 48, 863 (1983)] for the dipolar hard sphere fluid dielectric constant.

PACS number(s): 61.20.Gy, 77.22.Ch, 77.22.Ej

\section{INTRODUCTION}

A number of methods [2,3] have been used in theoretical and computer simulation studies of dielectric constant of polar fluids. However, all studies are based on the basic relation between the polarization $(\mathbf{P})$ and the electric field strength inside the dielectric $(\mathbf{E})$ :

$$
4 \pi \mathbf{P}=(\varepsilon-1) \mathbf{E},
$$

where $\varepsilon$ is the dielectric constant. Assuming we know the molecular parameters and the distribution functions, the polarization can be calculated for a bulk fluid phase. If we have the relation between the external field $\left(\mathbf{E}_{\text {ext }}\right)$ and $\mathbf{E}$, the dielectric constant can be expressed in terms of the density, temperature, and molecular quantities that appear in Eq. (1). In an ellipsoid-shaped dielectric, if the external field direction coincides with that of one of the principal axes, then the field strength inside the dielectric is

$$
\mathbf{E}=\frac{1}{1+\lambda(\varepsilon-1)} \mathbf{E}_{\mathrm{ext}},
$$

\footnotetext{
*Permanent address: Department of Physical Chemistry, University of Veszprém, H-8201 Veszprém, P.O. Box 158, Hungary. Electronic address: szalai@almos.vein.hu

†Electronic address: hrsccky@hku.hk

*Electronic address: doug@huey.byu.edu
}

where $\lambda$ is the depolarization factor. (The calculation of $\lambda$ is well known $[4,5]$.) For an infinitely prolate ellipsoid (needleshaped sample) $\lambda=0$, which means that

$$
\mathbf{E}=\mathbf{E}_{\mathrm{ext}} \text {. }
$$

For a dielectric with a spherical shape $\lambda=\frac{1}{3}$, the inside field strength (Maxwell field) becomes

$$
\mathbf{E}=\frac{3}{\varepsilon+2} \mathbf{E}_{\mathrm{ext}}
$$

For a dilute gas of dipolar particles, the polarization is given by the expansion of the Langevin function $[L(\alpha)]$ :

$$
\mathbf{P}=s \rho L(\alpha)=s \rho[\operatorname{coth}(\alpha)-1 / \alpha] \cong \frac{s^{2} \rho}{3 k T} \mathbf{E}_{\mathrm{ext}}
$$

where $s$ is the magnitude of the dipole moment, $\rho=N / V$ is the number density, $T$ is the temperature, $k$ is the Boltzmann constant, and $\alpha=s E_{\text {ext }} /(k T)$ is a dimensionless quantity. Using a spherically shaped sample, the Debye equation [6] for the dielectric constant can be obtained on the basis of Eqs. (1), (4), and (5) as

$$
\frac{\varepsilon-1}{\varepsilon+2}=y,
$$


where $y=4 \pi \rho s^{2} / 9 k T$ is the dimensionless dipole strength function. Considering the dipolar hard sphere (DHS) fluid, Jepsen $[7,8]$ and Rushbrooke $[9,10]$ have shown that the next exact term in the $y$ expansion of $(\varepsilon-1) /(\varepsilon+2)$ is given as

$$
\frac{\varepsilon-1}{\varepsilon+2}=y-\frac{15}{16} y^{2}
$$

Equation (7) gives correct third-order results in the $y$ expansion of the dielectric constant:

$$
\varepsilon=1+3 y+3 y^{2}+\frac{3}{16} y^{3}+\cdots .
$$

Note, that the $y$ expansion of Wertheim's [11] mean spherical approximation (MSA) dielectric constant also gives the above result. Onsager's [12] and Van Vleck's [13] theories are correct only up to second order in $y$. Using the Kirkwood equation [14], Tani et al. [15] obtained a perturbation theory equation for the dielectric constant of DHS fluid, which is

$$
\varepsilon=1+3 y+3 y^{2}+3 y^{3}\left(\frac{9 I_{d d \Delta}(\rho)}{16 \pi^{2}}-1\right) .
$$

Here $I_{d d \Delta}(\rho)$ is a density-dependent integral (see later), which can be calculated by the hard sphere (HS) correlation functions. In the low density limit $I_{d d \Delta}=17 \pi^{2} / 9$, and therefore Eq. (9) is identical to Eq. (8).

Recently, using the algebraic perturbation technique of Ruelle [16], Kalikmanov [1] predicted an equation for the dielectric constant of the DHS fluid. In his method, the external electric field is treated as a perturbation and the fluid sample is confined into an (infinitely) long cylinder with an axis parallel to the external field to avoid the depolarization. The polarization is calculated on the basis of free energy route, and the dielectric constant is obtained using Eqs. (1) and (3). In the low density approximation his result is

$$
\varepsilon=1+3 y+3 y^{2}+\frac{45}{16} y^{3} .
$$

This result is not in agreement with Eq. (8), which can be considered exact at low density up to the third-order term in the dipole strength function. The dielectric constant does not depend on the shape of the sample, therefore the difference between Eqs. (10) and (8) is not reasonable. Though the calculation route differs from the previous methods, the difference in the third-order terms is too high.

In this Comment we show that there are two errors in Kalikmanov's work in the calculation of the three-body terms of the field-dependent free energy and, therefore, his theory seems to be incomplete. Calculating these terms correctly, in the low density limit we can recover Eq. (8). Taking into account the density dependence of the correlation functions, our previous equation for the dielectric constant of DHS fluid [15] is obtained.

\section{THEORETICAL APPROACH}

\section{A. Model}

The potential energy of the $i j$ th pair of the DHS particles in an external field is

$$
\begin{aligned}
u\left(\mathbf{r}_{i j}, \omega_{i}, \omega_{j}\right)= & u_{d}\left(r_{i j}\right)-\frac{s^{2}}{r_{i j}^{3}} D(\mathbf{i}, \mathbf{j}) \\
& -s E_{\mathrm{ext}} \cos \vartheta_{i}-s E_{\mathrm{ext}} \cos \vartheta_{j}
\end{aligned}
$$

On the right side of Eq. (11) the first term is the hard sphere, the second is the dipole-dipole interaction energy, and the last two terms are the external field contributions. This notation is in accordance with [1] but for $D(\mathbf{i}, \mathbf{j})$ dipole-dipole orientation function, we use the most general expression

$$
D(\mathbf{i}, \mathbf{j})=3\left(\hat{\mathbf{s}}_{i} \cdot \hat{\mathbf{r}}_{i j}\right)\left(\hat{\mathbf{s}}_{j} \cdot \hat{\mathbf{r}}_{i j}\right)-\left(\hat{\mathbf{s}}_{i} \cdot \hat{\mathbf{s}}_{j}\right),
$$

which has the opposite sign of the corresponding Kalikmakov function. Without any further details, the second-order expression for the DHS free energy $(F)$ in the external field is

$$
\beta F=\beta F_{0}-\left(\frac{\rho}{4 \pi}\right) b_{1}-\frac{1}{2}\left(\frac{\rho}{4 \pi}\right)^{2} b_{2}
$$

where $F_{0}$ is the free energy of the reference system (DHS fluid without the external field) and $b_{i}$ is the $i$ th fielddependent perturbation term, which can be calculated with the help of the correlation functions of DHS fluid. The corresponding equations are

$$
b_{1}=\int d \mathbf{r}_{1} d \omega_{1} f\left(\omega_{1}\right)
$$

$$
b_{2}=\int d \mathbf{r}_{1} d \mathbf{r}_{2} d \omega_{1} d \omega_{2} f\left(\omega_{1}\right)\left[g_{2}^{0}\left(\mathbf{r}_{12}, \omega_{1}, \omega_{2}\right)-1\right] f\left(\omega_{2}\right),
$$

and

$$
f\left(\omega_{i}\right)=\exp \left(\alpha \cos \vartheta_{i}\right)-1 \cong \alpha \cos \vartheta_{i}+\left(\alpha^{2} / 2\right) \cos ^{2} \vartheta_{i} .
$$

To be consistent in the calculation of $b_{1}$, the second-order expansion of the Mayer function [Eq. (16)] must be used while in the calculation of $b_{2}$, a first-order expansion may be used. The exact DHS pair correlation function is not known, therefore in [1] a second-order perturbation expression was used [17]:

$$
\begin{aligned}
g_{2}^{0}\left(\mathbf{r}_{12}, \omega_{1}, \omega_{2}\right)= & g_{d}\left(r_{12}\right)+\left(\beta s^{2}\right) g_{1}\left(\mathbf{r}_{12}, \omega_{1}, \omega_{2}\right) \\
& +\left(\beta s^{2}\right)^{2} g_{2}\left(\mathbf{r}_{12}, \omega_{1}, \omega_{2}\right),
\end{aligned}
$$

where $g_{d}\left(r_{12}\right)$ is the HS pair correlation function, and the perturbation terms are

$$
g_{1}=\frac{g_{d}\left(r_{12}\right)}{r_{12}^{3}} D(\mathbf{1 , 2})
$$

$$
\begin{aligned}
g_{2}= & \frac{1}{2} D^{2}(\mathbf{1}, \mathbf{2}) \frac{g_{d}\left(r_{12}\right)}{r_{12}^{6}}-\frac{1}{6} \rho D(\mathbf{1}, \mathbf{2}) a_{D}\left(r_{12}\right) g_{d}\left(r_{12}\right) \\
& +\frac{1}{3} \rho \Delta(\mathbf{1 , 2}) a_{\Delta}\left(r_{12}\right) g_{d}\left(r_{12}\right)
\end{aligned}
$$


with $\Delta(\mathbf{1 , 2})=\hat{\mathbf{s}}_{1} \cdot \hat{\mathbf{s}}_{2}$ and Kirkwood's superposition approximation used for the three-body correlation function. In Eq. (19) we introduce two functions with the following definitions:

$$
\begin{gathered}
a_{D}\left(r_{12}\right)=\int d \mathbf{r}_{3} \frac{1+3 \cos \alpha_{1} \cos \alpha_{2} \cos \alpha_{3}}{\left(r_{13} r_{23}\right)^{3}} g_{d}\left(r_{13}\right) g_{d}\left(r_{23}\right), \\
a_{\Delta}\left(r_{12}\right)=\int d \mathbf{r}_{3} \frac{3 \cos ^{2} \alpha_{3}-1}{\left(r_{13} r_{23}\right)^{3}} g_{d}\left(r_{13}\right) g_{d}\left(r_{23}\right) .
\end{gathered}
$$

\section{B. Low-density limit}

First we study the low-density approximation, which means that in Eqs. (17)-(19) the HS pair correlation functions are replaced by the Heaviside step function $\left[g_{d}(r)\right.$ $=\Theta(r-d)]$, where $d$ is the HS diameter. In accordance with [1] we can obtain that

$$
b_{1}=4 \pi \frac{\alpha^{2}}{6} V .
$$

According to the three-term expansion of $g_{2}^{0}\left(\mathbf{r}_{12}, \omega_{1}, \omega_{2}\right)$, Kalikmanov separated $b_{2}$ into three terms:

$$
b_{2}=\alpha^{2} V\left(b_{2}^{(0)}+b_{2}^{(1)}+b_{2}^{(2)}\right) .
$$

It is easy to see that $b_{2}^{(0)}=0$. Using the solid angle integration

$$
\begin{gathered}
\int d \omega_{1} d \omega_{2} \cos \vartheta_{1} D(\mathbf{1 , 2}) \cos \vartheta_{2} \\
=-\left(\frac{4 \pi}{3}\right)^{2}\left(1-3 \cos ^{2} \vartheta_{12}\right)
\end{gathered}
$$

we obtain

$$
b_{2}^{(1)}=-\beta s^{2}\left(\frac{4 \pi}{3}\right)^{2} \int_{r_{12}>d} d \mathbf{r}_{12} \frac{1}{r_{12}^{3}}\left(1-3 \cos ^{2} \vartheta_{12}\right) .
$$

In the calculation of the integral of Eq. (25), Kalikmanov assumed that the container is an (infinitely) long cylinder with an axis parallel to the external field. It is better to mention an infinitely prolate ellipsoid (needle-shaped sample) because in this case the depolarization factor is exactly zero. The calculation of the integral in Eq. (25) is not trivial for such a shape, but Groh and Dietrich $[18,19]$ have predicted a relatively simple method for that. Using this method we obtain

$$
b_{2}^{(1)}=\beta s^{2}\left(\frac{4 \pi}{3}\right)^{3}
$$

which is identical with the result of [1]. The first term in Eq. (19) is really short ranged and therefore in this approximation, this term does not give any contribution to the fielddependent free energy.

There are problems in [1] with the calculation of $b_{2}^{(2)}$. The function $a_{D}\left(r_{12}\right)$ is long ranged and therefore gives a very important contribution to the field-dependent free energy. The role of this term was ignored in Kalikmanov's work. The function $a_{\Delta}\left(r_{12}\right)$ is really short ranged but the calculation of its integral is not correct in Kalikmakov's paper. The $a_{D}\left(r_{12}\right)$ and $a_{\Delta}\left(r_{12}\right)$ functions are convolution integrals and with the help of the Fourier-transformconvolution method of Høye and co-workers [20,21], also used by Goldman [22], they can be calculated relatively easily. The details of the calculation can be found in the Appendix. In the low-density limit the following exact relations are obtained:

$$
a_{D}\left(r_{12}\right)=\frac{1}{d^{3}} \times \begin{cases}\frac{\pi}{12}\left[-\left(\frac{r_{12}}{d}\right)^{3}+6\left(\frac{r_{12}}{d}\right)\right] & \text { if } \frac{r_{12}}{d}<2 \\ \frac{8 \pi}{3}\left(\frac{d}{r_{12}}\right)^{3} & \text { if } \frac{r_{12}}{d} \geqslant 2,\end{cases}
$$

$a_{\Delta}\left(r_{12}\right)=\frac{1}{d^{3}} \times\left\{\begin{array}{l}\frac{\pi}{12}\left[2\left(\frac{r_{12}}{d}\right)^{3}-24\left(\frac{r_{12}}{d}\right)+32\right] \quad \text { if } \frac{r_{12}}{d}<2 \\ 0 \quad \text { if } \frac{r_{12}}{d} \geqslant 2 .\end{array}\right.$

Using these functions and Eq. (24) for $b_{2}^{(2)}$ we obtain

$$
\begin{aligned}
b_{2}^{(2)}= & \left(\beta s^{2}\right)^{2} \frac{\rho}{6} \frac{16 \pi^{2}}{9} \int d \mathbf{r}_{12} a_{D}\left(r_{12}\right) \\
& \times \Theta\left(r_{12}-d\right)\left(1-3 \cos ^{2} \vartheta_{12}\right) \\
& +\left(\beta s^{2}\right)^{2} \frac{\rho}{3} \frac{16 \pi^{2}}{9} \int d \mathbf{r}_{12} a_{\Delta}\left(r_{12}\right) \Theta\left(r_{12}-d\right),
\end{aligned}
$$

where we took into account that

$$
\int d \omega_{1} d \omega_{2} \cos \vartheta_{1} \Delta(\mathbf{1}, \mathbf{2}) \cos \vartheta_{2}=\frac{16 \pi^{2}}{9}
$$

Equation (27) shows that $a_{D}\left(r_{12}\right)$ is long ranged and, therefore, in the case of the first integral of Eq. (29), the integration must be carried out on an infinitely prolate ellipsoid. Using the method of Groh and Dietrich $[18,19]$ we obtain

$$
\begin{aligned}
\int & d \mathbf{r}_{12}\left[a_{D}\left(r_{12}\right) \Theta\left(r_{12}-d\right)\left(1-3 \cos ^{2} \vartheta_{12}\right)\right] \\
= & \frac{8 \pi}{3} \int_{r_{12}>d} d \mathbf{r}_{12} \frac{1}{r_{12}^{3}}\left(1-3 \cos ^{2} \vartheta_{12}\right) \\
= & -\frac{32 \pi^{2}}{9} .
\end{aligned}
$$

This result is not surprising, because their equation shows [Eq. (3.19) in Ref. [18]] that only the asymptotic limit of the $a_{D}\left(r_{12}\right)$ function gives a contribution to the integral.

We can see in Eq. (28) that $a_{\Delta}\left(r_{12}\right)$ is really short ranged, which makes it possible to replace its integration over an ellipsoid by an integration over a sphere: 


$$
\int d \mathbf{r}_{12} a_{\Delta}\left(r_{12}\right) \Theta\left(r_{12}-d\right)=4 \pi \int_{d}^{2 d} d r_{12} a_{\Delta}\left(r_{12}\right) r_{12}^{2}=\frac{17 \pi^{2}}{9} .
$$

It is easy to see that the integral in Eq. (32) is the low-density limit of the integral introduced by Tani et al. [15]:

$$
\begin{aligned}
I_{d d \Delta}(\rho=0)= & \int d \mathbf{r}_{12} a_{\Delta}\left(r_{12}\right) \Theta\left(r_{12}-d\right) \\
= & \int d \mathbf{r}_{12} \Theta\left(r_{12}-d\right) \int d \mathbf{r}_{3} \frac{3 \cos ^{2} \alpha_{3}-1}{r_{13}^{3} r_{23}^{3}} \\
& \times \Theta\left(r_{13}-d\right) \Theta\left(r_{23}-d\right) .
\end{aligned}
$$

Kalikmanov obtained $5 \pi^{2} / 3$ for this integral, which is not correct. He cited the Appendix of one of his earlier publications [23], where he calculated that integral. However, in [23] there is an error in the conversion of integration variables and, therefore, in [24] he calculated a completely different integral. In Eq. (33) the volume element $d \mathbf{r}_{3}$ can be considered as that of a sphere around particle 1, i.e., $d \mathbf{r}_{3}$ $=-r_{13}^{2} d r_{13} d\left(\cos \alpha_{3}\right) d \varphi$ [23], where $\varphi$ is the azimuthal angle extending over $2 \pi$. After integration over the possible angles it reads

$$
\begin{aligned}
I_{d d \Delta}= & -8 \pi^{2} \int_{d}^{\infty} d r_{12} r_{12}^{2} \int_{d}^{\infty} d r_{13} r_{13}^{2} \int_{-1}^{1} d\left(\cos \alpha_{3}\right) \\
& \times \frac{3 \cos ^{2} \alpha_{3}-1}{r_{13}^{3} r_{23}^{3}} \Theta\left(r_{23}-d\right),
\end{aligned}
$$

which is identical with the formula of Tani et al. [15] but completely different from the corresponding results in [1] and [23].

Summarizing all calculated terms, for the free energy we obtain

$$
\begin{aligned}
\beta F= & \beta F_{0}-\frac{1}{6} V \rho \alpha^{2}-\frac{4 \pi}{54} V \rho^{2} \beta s^{2} \alpha^{2} \\
& +\frac{8 \pi^{2}}{243} V \rho^{3}\left(\beta s^{2}\right)^{2} \alpha^{2}-\frac{17 \pi^{2}}{486} V \rho^{3}\left(\beta s^{2}\right)^{2} \alpha^{2} .
\end{aligned}
$$

The polarization can be calculated from Eq. (35) as

$$
\mathbf{P}=-\frac{1}{V}\left(\frac{\partial F}{\partial \mathbf{E}_{\mathrm{ext}}}\right)_{N, V, T} .
$$

Using the dipole strength function for the polarization we obtain

$$
\mathbf{P}=\frac{3}{4 \pi}\left(y+y^{2}-y^{3}+\frac{17}{16} y^{3}\right) \mathbf{E}_{\mathrm{ext}} .
$$

From the polarization, the dielectric constant can be calculated by the help of Eqs. (1) and (3). Hence,

$$
\varepsilon=1+3 y+3 y^{2}+\frac{3}{16} y^{3},
$$

which is in agreement with the formula of Tani et al. [15] obtained by a different route.

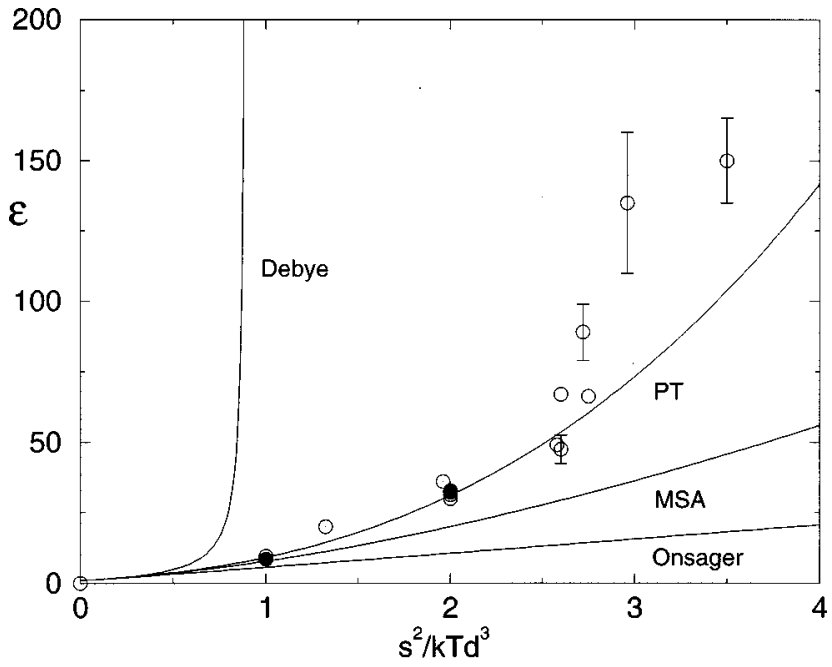

FIG. 1. Dielectric constant as a function of $s^{2} / k T d^{3}$ for $\rho d^{3}$ $=0.8$. Open circles denote simulation results in Ref. [1]. Filled circles are our simulation data from Refs. [25,26]. PT refers to our perturbation calculations.

\section{Density dependence of dielectric constant}

The dielectric constant of DHS fluid depends on the density, beside the dipole strength function $[2,3,15]$. To obtain the functional form, the correct density-dependent $g_{d}(r, \rho)$ must be used to calculate the integrals that appear. In this case for $b_{2}^{(1)}$ we obtain

$$
b_{2}^{(1)}=-\beta s^{2}\left(\frac{4 \pi}{3}\right)^{2} \int_{r_{12}>d} d \mathbf{r}_{12} \frac{g_{d}\left(r_{12}, \rho\right)}{r_{12}^{3}}\left(1-3 \cos ^{2} \vartheta_{12}\right) .
$$

On the basis of the results of Groh and Dietrich $[18,19]$ it is clear that only the asymptotic values of $g_{d}\left(r_{12}, \rho\right) / r_{12}^{3}$ give contributions to the integral. Since the asymptotic limit of this function is $1 / r_{12}^{3}$, the result of the integration on an infinitely prolate ellipsoid is the same that was obtained in Eq. (26). Considering the $b_{2}^{(2)}$ term, the obtained asymptotic value of the density-dependent $a_{D}\left(r_{12}, \rho\right)$ function is the same as was the density-independent one (see the Appendix); therefore the numerical value of the corresponding integral does not change. Only the integral of the short-ranged $a_{\Delta}\left(r_{12}, \rho\right)$ function shows a density dependence. This integral can be calculated on a sphere, and according to Eq. (21) that corresponds to the $I_{d d \Delta}(\rho)$ integral of Tani et al. [15]. Of course the derived equation for the dielectric constant is the same as given by Eq. (9). According to the abovementioned arguments, Kalikmanov's corresponding equation for the dielectric constant is incorrect. Therefore, the comparison with simulation data (Fig. 1 in Ref. [1]) is inappropriate. Of course, his results for the initial magnetic susceptibility of a ferrofluid (Fig. 2 in Ref. [1]) must be recalculated again. The reduced dipole moment dependence of our formulated dielectric constant is displayed in Fig. 1. At low and moderate dipole moments, the agreement with simulation data is quite good. The theory prediction is below the simulation data at high dipole moments when $s^{2} / k T d^{3}$ $\geqslant 2.5$. To help the concrete calculations, on the basis of [15] here we give again the Padé-type fitted expression for the $I_{d d \Delta}$ integral: 


$$
I_{d d \Delta}\left(\rho^{*}\right)=\frac{17 \pi^{2}}{9} \frac{1-0.93952 \rho^{*}+0.36714 \rho^{* 2}}{1-0.92398 \rho^{*}+0.23323 \rho^{* 2}},
$$

where $\rho^{*}=\rho d^{3}$ is the reduced density, and Eq. (40) is valid in the range of $0 \leqslant \rho^{*} \leqslant 0.95$. Further applications to the calculation of dielectric constant of hard-core dipolar Yukawa fluid have been published recently $[25,26]$ by us.

\section{SUMMARY}

To study the dielectric constant of DHS fluids, Kalikmanov [1] used Ruelle's [16] algebraic perturbation theory in the construction of the field-dependent free energy. It has been shown that with a correct calculation of the three-body term integrals this free energy route gives consistent results for the dipole strength and density dependence of the dielectric constant. In spite of the fact that these results are known $[15,22,26]$, the improved method should be very valuable in the study of thermodynamic properties of fluids in an external field. As there is nothing special in the theory that restricts it to electric dipoles, it is valid for the description of a magnetic colloid suspension in an external magnetic field.

\section{ACKNOWLEDGMENTS}

This research was supported in part by the Hungarian National Research Fund (OTKA-TO25884) and the U.S. NSF (Grant No. CHE98-13729). K.Y.C. also thanks the University of Hong Kong for financial support and the Research Grants Council of Hong Kong for Grant No. HKU 7213199P

\section{APPENDIX}

To calculate the functional forms of $a_{D}\left(r_{12}\right)$ and $a_{\Delta}\left(r_{12}\right)$ integrals, the Fourier-transform-convolution method [20-22] is applied. Both functions are convolution integrals; therefore following Høye and Stell [21] by introducing the

$$
f(r)=\frac{g_{d}(r)}{r^{3}}
$$

function we can obtain the following equations:

$$
\begin{aligned}
& a_{D}(r)=\frac{1}{\pi^{2}} \int_{0}^{\infty} d k j_{2}(k r)[\bar{f}(k)]^{2} k^{2}, \\
& a_{\Delta}(r)=\frac{1}{\pi^{2}} \int_{0}^{\infty} d k \frac{\sin (k r)}{(k r)}[\bar{f}(k)]^{2} k^{2} .
\end{aligned}
$$

Here $\bar{f}(k)$ is not the Fourier transform but a Hankel transform:

$$
\bar{f}(k)=-4 \pi \int_{0}^{\infty} d r j_{2}(k r) f(r) r^{2},
$$

with the second-order Bessel function:

$$
j_{2}(x)=\frac{3 \sin x}{x^{3}}-\frac{3 \cos x}{x^{2}}-\frac{\sin x}{x} .
$$

On the basis of the work of Høye and Stell [21] for the asymptotic limit of $a_{D}\left(r_{12}\right)$ function we can write

$$
a_{D}\left(r_{12}\right) \underset{r_{12} \rightarrow \infty}{\sim} \frac{3[\bar{f}(0)]^{2}}{2 \pi r_{12}^{3}}=\frac{8 \pi}{3 r_{12}^{3}},
$$

which is of course independent of the density.

Note that our formulas are not the same as the equations of Høye and Stell [21]. In our definition of $a_{\Delta}\left(r_{12}\right)$, there is an extra factor of 3 in Eq. (21) while in the definition of $a_{D}\left(r_{12}\right)$ an extra factor of $(-6)$ is in Eq. (20). The first factor is obvious, while the signature of the second one comes from the fact that in Høye and Stell's $A_{D}$ function (Eqs. C6 and C7 in Ref. [21]), one angle in the triangle of $(1,2,3)$ particles is a supplementary angle and therefore its cosine is negative.
[1] V. I. Kalikmanov, Phys. Rev. E 59, 4085 (1999).

[2] G. Stell, G. N. Patey, and J. S. Høye, Adv. Chem. Phys. 48, 183 (1981).

[3] S. W. de Leeuw, J. W. Perram, and E. R. Smith, Annu. Rev. Phys. Chem. 37, 245 (1986).

[4] B. K. P. Scaife, Principles of Dielectrics, 2nd ed. (Clarendon Press, Oxford, 1998), Chap. 2.

[5] J. A. Stratton, Electromagnetic Theory, 1st ed. (McGraw-Hill, New York, 1941), Chap. 3.

[6] P. Debye, Phys. Z. 13, 97 (1912).

[7] D. W. Jepsen, J. Chem. Phys. 44, 774 (1966).

[8] D. W. Jepsen, J. Chem. Phys. 45, 709 (1966).

[9] G. S. Rushbrooke, Mol. Phys. 37, 761 (1979).

[10] G. S. Rushbrooke, Mol. Phys. 43, 975 (1981).

[11] M. S. Wertheim, J. Chem. Phys. 55, 4291 (1971).

[12] L. Onsager, J. Am. Chem. Soc. 58, 1486 (1936).

[13] J. H. Van Vleck, J. Chem. Phys. 5, 556 (1937).

[14] J. G. Kirkwood, J. Chem. Phys. 7, 911 (1939).

[15] A. Tani, D. Henderson, J. A. Barker, and C. E. Hecht, Mol.
Phys. 48, 863 (1983).

[16] D. Ruelle, Statistical Mechanics: Rigorous Results (Benjamin, New York, 1969).

[17] J. A. Barker and D. Henderson, Rev. Mod. Phys. 48, 587 (1976).

[18] B. Groh and S. Dietrich, Phys. Rev. E 50, 3814 (1994).

[19] B. Groh and S. Dietrich, Phys. Rev. E 53, 2509 (1996).

[20] J. S. Høye, J. L. Lebowitz, and G. Stell, J. Chem. Phys. 61, 3253 (1974).

[21] J. S. Høye and G. Stell, J. Chem. Phys. 63, 5342 (1975).

[22] S. Goldman, Mol. Phys. 71, 491 (1990).

[23] V. I. Kalikmanov, Physica A 183, 25 (1992).

[24] K. Lucas, Applied Statistical Thermodynamics (SpringerVerlag, Berlin, 1991), p. 307.

[25] D. Henderson, D. Boda, I. Szalai, and K. Y. Chan, J. Chem. Phys. 110, 7348 (1999).

[26] I. Szalai, D. Henderson, D. Boda, and K. Y. Chan, J. Chem. Phys. 111, 337 (1999). 